\title{
The Conservation of Matter in General Relativity
}

\author{
STEPHEN HAWKing \\ Institute of Theoretical Astronomy, Cambridge, England
}

Received June 1, 1970

\begin{abstract}
It is shown that in classical general relativity, if space-time is nonempty at one time, it will be nonempty at all times provided that the energy momentum tensor of the matter satisfies a physically reasonable condition. The apparent contradiction with the quantum predictions for the creation and annihilation of matter particles by gravitons is discussed and is shown to arise from the lack of a good energy momentum operator for the matter in an unquantised curved space-time metric.
\end{abstract}

\section{Introduction}

In the general theory of relativity it is well known that the equations $T^{a b}{ }_{; b}=0$, which express the local conservation of energy and momentum, cannot be integrated to give conservation laws over a region. This is because the tensor $T^{a b}$ represents the energy-momentum only of matter fields and not of the gravitational field. It is a matter of common experience that the energy of a system is not conserved unless one also takes into account its gravitational energy. In Newtonian theory the concept of gravitational energy is well defined but in general relativity this is unfortunately not the case for arbitrary fields. However, for a bounded system in an asymptotically flat space-time one can define a total energy or mass which represents the energy of both the matter and the gravitational field and which decreases at a rate which can be interpreted as the rate at which energy is carried away to infinity by gravitational radiation [1-4]. The question then arises: could the system radiate away all its mass as gravitational waves and leave just empty space? It will be shown that it could not in the classical theory if $T^{a b}$ satisfies a physically reasonable condition. That is to say a space-time which is non-empty at one time must be non-empty at all times and conversely one which is empty at one time must be empty at all times. The proof depends only on the equations $T^{a b}{ }_{; b}=0$ and not on the field equations.

This result would seem to be in contradiction with the non-zero cross-sections which have been calculated for such processes as the annihilation of a pair of particles into gravitons (see, for example [5]). The reason for this discrepancy between classical and quantum theories 
are discussed in Section 3. It seems to be related to the difficulty of defining a local energy-momentum operator for the matter fields in a curved space-time.

\section{Classical Theory}

The energy-momentum tensor will be said to satisfy the dominant energy condition if for every observer the local energy density $T_{a b} U^{a} U^{b}$ is non-negative and the local energy flow vector $T_{a b} U^{a}$ is non-spacelike where $U^{a}$ is the velocity vector of the observer $\left(U^{a} U_{a}>0\right)$. The first of these would seem to be required by local quantum mechanics and the second by causality. They are satisfied by all known forms of matter. With a bit of algebra the dominant energy condition can be shown to be equivalent to the requirement that in any orthonormal tetrad the energy component $T_{00}$ should be greater than or equal to the absolute values of the other components of the energy-momentum tensor i.e.

$$
T_{00} \geqq\left|T_{a b}\right| \quad \text { for each } a, b \text {. }
$$

For a fluid with energy density $\mu$ and principal pressures $p_{i}(i=1,2,3)$ this will hold if and only if $\mu \geqq\left|p_{i}\right|$. This is a very reasonable requirement since if the pressure were to exceed the energy density, there would be sound waves which travelled faster than light.

We shall show that if the dominant energy condition holds, a region which is empty initially remains empty provided no matter flows in from outside. To be more precise we shall show that $T_{a b}$ vanishes in a compact region $F$ whose boundary $\partial F$ consists of a part $(\partial F)_{1}$ on which $T_{a b}$ vanishes and a part $(\partial F)_{2}$ whose outward normal is future directed and timelike or null. We shall assume that there is a function $t$ whose gradient is everywhere future directed and timelike. Such a function will exist provided space-time is not on the verge of violating causality [6].

Let $H\left(t^{\prime}\right)$ denote the spacelike surface $t=t^{\prime}$ and let $F\left(t^{\prime}\right)$ denote the part of $F$ to the past of $H\left(t^{\prime}\right)$. Consider the volume integral

$$
I(t)=\int_{F(t)}\left(T^{a b} t_{; a}\right)_{; b} d v=\int_{F(t)} T^{a b} t_{; a b} d v
$$

where $d v=\sqrt{-g} d^{4} x$ is the invariant volume element. By Gauss's theorem this can be transformed into an integral over the boundary of $F(t)$,

$$
I(t)=\int_{\partial F(t)} T^{a b} t_{; a} d S_{b}
$$


The boundary of $F(t)$ will consist of $F(t) \cap(\partial F)_{1}, F(t) \cap(\partial F)_{2}$ and $F \cap H(t)$. Since $T_{a b}$ is zero on $(\partial F)_{1}$,

$$
I(t)=\int_{F(t) \cap(\partial F)_{2}}+\int_{F \cap H(t)} .
$$

By the dominant energy condition the first term on the right is nonnegative since the outward normal to $(\partial F)_{2}$ is future directed. Thus

$$
I(t)=\int_{F(t)} T^{a b} t_{; a b} d v \geqq J(t)=\int_{F \cap H(t)} T^{a b} t_{; a} d S_{b} .
$$

Since $F$ is compact there will be some upper bound to the components of $t_{; a b}$ in any orthonormal tetrad whose timelike vector is in the direction of $t_{; a}$. Thus, by the dominant energy condition there will be some $C>0$ such that on $F$

$$
T^{a b} t_{; a b} \leqq C T^{a b} t_{; a} t_{; b} .
$$

The integral over $F(t)$ can be decomposed into an integral over the surfaces $H\left(t^{\prime}\right)$ followed by an integral with respect to $t^{\prime}$ :

$$
I(t) \leqq C \int^{t}\left(\int_{F \cap H\left(t^{\prime}\right)} T^{a b} t_{; a} d S_{b}\right) d t^{\prime} .
$$

Therefore $J(t) \leqq C \int^{t} J\left(t^{\prime}\right) d t^{\prime}$.

But for sufficiently early values of $t, H(t)$ will not intersect $F$ and so $J(t)$ will vanish. Thus $J(t)$ will vanish for all $t$ which implies that $T^{a b}$ vanishes on $F$.

In other words, if space-time is empty at one time, then it will remain empty providing that matter does not come in from infinity. Conversely, a bounded system which is present at one time cannot fail to be present at all other times.

\section{Quantum Theory}

To investigate the apparent contradiction between the result of the previous section and the nonzero cross-sections which have been calculated for the creation or annihilation of particles by gravitons we shall consider the case of a Hermitian scalar field $\phi$ of mass $m$ which obeys the covariant Klein-Gordon equation

$$
g^{a b} \phi_{; a b}+m^{2} \phi=0 .
$$

As the field equations were not used in previous sections, we shall take the metric to be a given external (unquantised) field unrelated to any matter content. For simplicity we shall consider a space-time consisting 
of initial and final flat space regions $M_{1}$ and $M_{3}$ separated by a region $M_{2}$ in which the curvature is nonzero. In $M_{1}$ and $M_{3}$ the operator $\phi$ behaves as that of a free field and may be given the standard quantum field theory interpretation. We may decompose $\phi$ into $\phi_{1}^{+}$which has only positive frequencies on $M_{1}$ and $\phi_{1}^{-}$which has only negative frequencies on $M_{1}$ i.e.

$$
\begin{aligned}
\phi(x) & =\phi_{1}^{+}(x)+\phi_{1}^{-}(x) \\
& =\sum_{i}\left(f_{1 i}(x) a_{i}+\bar{f}_{1 i}(x) a_{i}^{*}\right)
\end{aligned}
$$

where the $a_{i}$ are operators independent of position and the $f_{1 i}$ are a complete orthonormal set of complex functions which satisfy the covariant Klein-Gordon equation and contain only positive frequencies on $M_{1}$. Similarly $\phi$ may be decomposed into $\phi_{3}^{+}$and $\phi_{3}^{-}$, its positive and negative frequency parts on $M_{3}$. However, because of the intervening curvature in the region $M_{2}, \phi_{3}^{+}(x)$ will not in general be equal to $\phi_{1}^{+}(x)$. In other words, a solution $f_{1 i}$ of the covariant Klein-Gordon equation which has only positive frequency components on $M_{1}$ may have a negative frequency component on $M_{3}$. It is this intermixing of the positive and the negative frequencies which is responsible for the creation or annihilation of particles by the gravitational field. For in $M_{1}, \phi_{1}^{+}(x)$ and $\phi_{1}^{-}(x)$ may be interpreted as the operators which respectively annihilate and create a particle at the point $x$. Thus the condition on the state-vector $\mid>$ that there should be no particles in $M_{1}$ is not that $\phi(x) \mid>=0$ but that $\phi_{1}^{+}(x) \mid>=0$ for all $x$ in $M_{1}$. By the covariant Klein-Gordon equation $\phi_{1}^{+}(x) \mid>$ will then also be zero for $x$ in $M_{2}$ or $M_{3}$. However the particle annihilation operator in $M_{3}$ is $\phi_{3}^{+}(x)$ and because of the frequency mixing, $\phi_{3}^{+}(x) \mid>$ may be nonzero corresponding to a nonzero probability of finding a particle at the point $x$ in $M_{3}$. For any reasonable gravitational field this probability will be very low. Parker [7] has calculated that the rate of production of $\pi_{0}$ mesons by the gravitational field of the universe is about one particle a second in the whole universe.

The energy-momentum tensor for a classical scalar field $\phi$ satisfying the covariant Klein-Gordon equation is

$$
T_{a b}=\phi_{; a} \phi_{; b}+\frac{1}{2} g_{a b}\left(m^{2} \phi^{2}-\phi_{; c} \phi_{; d} g^{c d}\right) .
$$

This obeys $T^{a b}{ }_{; b}=0$ and satisfies the dominant energy condition. There is difficulty however in defining an energy-momentum operator in quantum theory. For if in the above expression one regards the $\phi$ 's as operators, one obtains an operator whose expection value even in flat, empty space is infinite. This is because there are negative frequency 
parts (creation operators) of $\phi$ standing to the right of positive frequency parts (annihilation operators). In flat space-time one way of overcoming this difficulty is to adopt normal ordering for products of $\phi$ 's i.e. decompose the $\phi$ 's into positive and negative frequency parts and rearrange the terms so that the negative frequencies are to the left and the positive to the right. However, as we have seen, this splitting into positive and negative frequency parts is not uniquely defined in curved space-time. Another possible way round the difficulty would be to try to define the energy momentum operator as the limit of a nonlocal operator in which the two $\phi$ operators are referred to different points and the vacuum expectation value is subtracted out. However this operator does not satisfy the covariant conservations $T_{; b}^{a b}=0$ in a general space-time. In fact there is no operator which satisfies these equations and has the right properties to be interpreted as the energy-momentum operator. For, if there were such an operator, one could use its expectation value in the theorem of the previous section and prove that it was zero on $M_{2}$ and $M_{3}$ if it was zero on $M_{1}$.

The cause of the difficulty seems to be the fact that we have quantised the matter fields but not the metric. This means that we have included the effect of the metric in creating or annihilating particles but have necessarily ommitted the reaction of these particles back on the metric i.e. the corresponding annihilation or creation of gravitons. To describe this properly would require a quantised theory of General Relativity which has not yet been developed in a completely satisfactory form (see [5] for a review). However, for most purposes, a classical treatment of the metric should be sufficient since quantum gravitational effects are probably important only when the radius of curvature is of the order of $10^{-33} \mathrm{cms}$. On the other hand one knows that a quantum theory is required for the matter fields to describe their local behaviour. What one wants therefore is a consistent approximation which treats the metric classically and the matter quantum mechanically. This can be obtained by assuming that the quantum operators for the matter fields obey the usual wave equations with the curved space-time metric in place of the Minkowski metric and taking the energy momentum tensor on the right of the classical Einstein equations to be the expectation value of the energy momentum operator defined from the classical expression by normal ordering where the splitting into positive and negative frequencies is chosen arbitrarily, for example that valid in the region $M_{1}$, say. This energy momentum tensor will not contain any contribution from particles created by the gravitational field. To the extent that such particles can be ignored (which is completely, except near a space-time singularity), this classical-quantum theory should be a good approximation. 


\section{References}

1. Bondi, H., van der Burg, M. G. J., Metzner, A. W. K.: Proc. Roy. Soc. A269, 21 (1962).

2. Newman, E. T., Unti, T. W. J.: J. Math. Phys. 3, 891 (1962).

3. Penrose, R.: Phys. Rev. Letters 10, 66 (1963).

4. Price, R., Thorne, K. S.: Astrophys. J. 155, 163 (1969).

5. DeWitt, B. S.: Phys. Rev. 160, 1113, 162, 1195, 162, 1239 (1967).

6. Hawking, S. W.: Proc. Roy. Soc. A 308, 433 (1968).

7. Parker, L.: Phys. Rev. Letters 21, 561 (1968).

S. W. Hawking Dept. of Applied Mathematics and Theoretical Physics University of Cambridge Silver Street

Cambridge/England 